\title{
Composition Analysis by EDS at Elevated Temperatures and More
}

\author{
Meiken Falke ${ }^{1 *}$ and Igor Nemeth ${ }^{1}$. \\ 1. Bruker Nano GmbH, Berlin, Germany. \\ *meiken.falke@bruker.com
}

We discuss possibilities and challenges of energy dispersive X-ray spectroscopy (EDS) and EDS element mapping at elevated temperatures in SEM and STEM. An electron transparent Pd-Aunanoparticle test structure on carbon film investigated in conventional STEM is one example. The agglomeration and evaporation of its nanoparticle material during heating from room temperature up to $1000^{\circ} \mathrm{C}$ were studied. Chemical phase analysis and simple statistical methods were applied to quantify the areal coverage of different species and its change with rising temperature. The stability of the heating holder in Z-direction during temperature change ensured very low drift and successful element mapping and phase tracking [1].

Heat radiation at elevated temperatures changes the low energy part of EDS spectra. We demonstrate, that element identification below $2 \mathrm{kV}$ and chemical phase analysis are still possible within certain limits. E.g. nitrogen can be detected up to $900^{\circ} \mathrm{C}$ (Fig.1). The results of phase and particle analysis (Fig.2) demonstrate the capabilities of in situ EDS in combination with statistical methods. Material agglomeration and evaporation of one involved species, the gold, during heating could be quantified by areal coverage values.

Further options of using EDS for in situ analysis are discussed. Speed of analysis defines, which processes can be usefully analyzed in situ by EDS. Optimum photon detection geometry is crucial for efficient, and thus fast, EDS in the electron microscope. Large solid and a high take-off angles for photon collection, proper collimation and suitable sample holders are desired. Multi-detector arrangements enable large solid angles at high take-off angles particularly well. The specimen holders should not shadow the detector(s). Holder and window materials and even reaction species sticking to them must be well known to ensure correct quantitative analysis of spectroscopic data, as e.g. in case of reaction cells holding liquid samples between electron transparent membranes. Just geometric optimization in combination with high brightness electron sources and aberration correction allows the identification of single atoms at low accelerating voltages in STEM within a few seconds during tracking [2]. This looks promising thinking of new challenging in situ experiments. Additionally, streams of constantly changing data require new ways of data acquisition and processing, which will be discussed.

\section{References:}

[1] T T van Omme et al., Ultramicroscopy 129 (2018) 14.

[2] R M Stroud et al. Appl. Phys. Lett. 108 (2016) 163101.

[3] The authors kindly acknowledge the support by Sander van Weperen from DENSsolutions (test sample, heating holder and data acquisition) and Holm Kirmse from Humboldt University (data acquisition on STEM). 

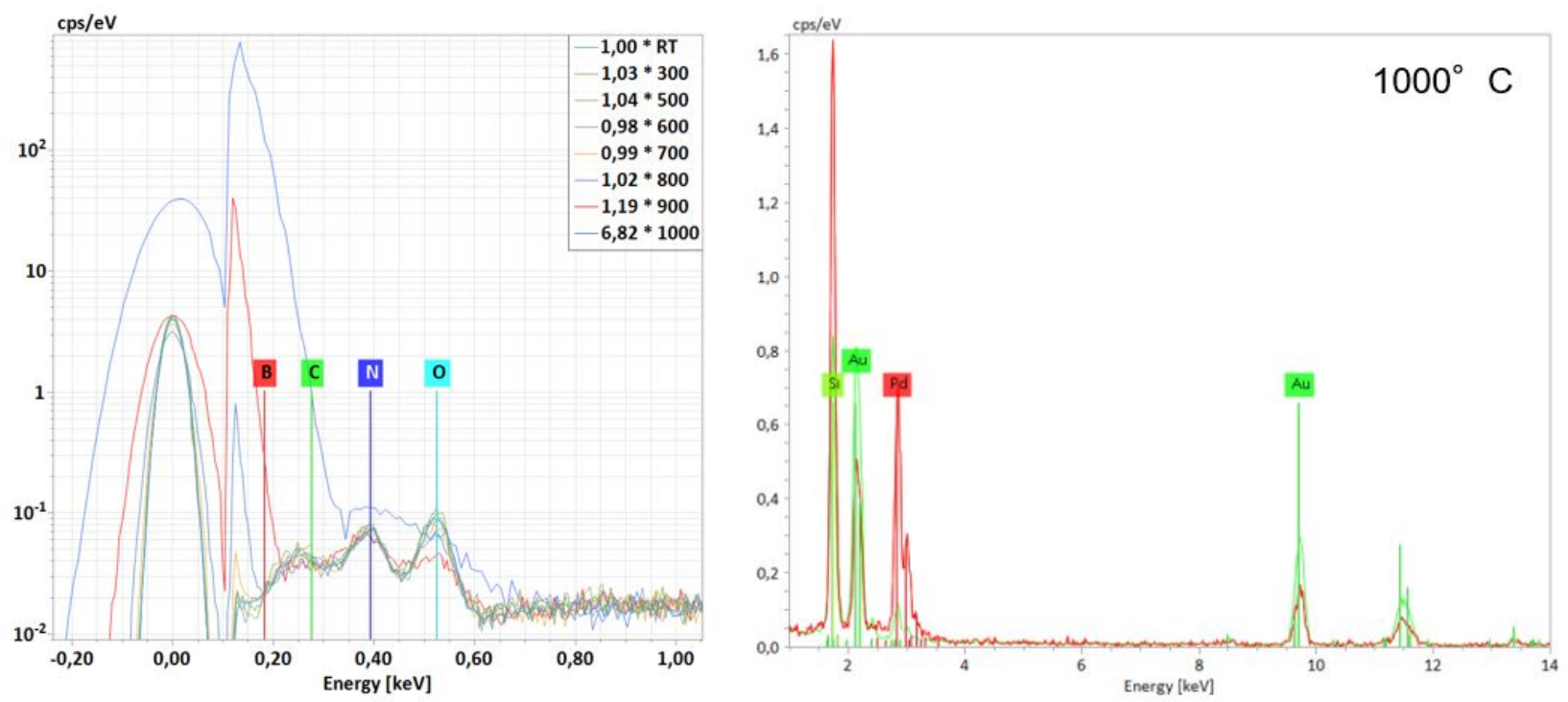

Figure 1. Left: The low energy part of EDS spectra from room temperature to $1000^{\circ} \mathrm{C}$ normalized to a background region around $1 \mathrm{kV}$. Nitrogen (part of the substrate) can be identified up to $900^{\circ} \mathrm{C}$. At $1000^{\circ} \mathrm{C}$ the elevated background intensity obscures the oxygen signal; Right: Spectra of the two phases of the test structure, $\mathrm{Au}=\mathrm{P} 2$ (green) and $\mathrm{Pd}=\mathrm{P} 3$ (red) at $1000^{\circ} \mathrm{C}$. $\mathrm{Si}$ is part of the specimen holder.
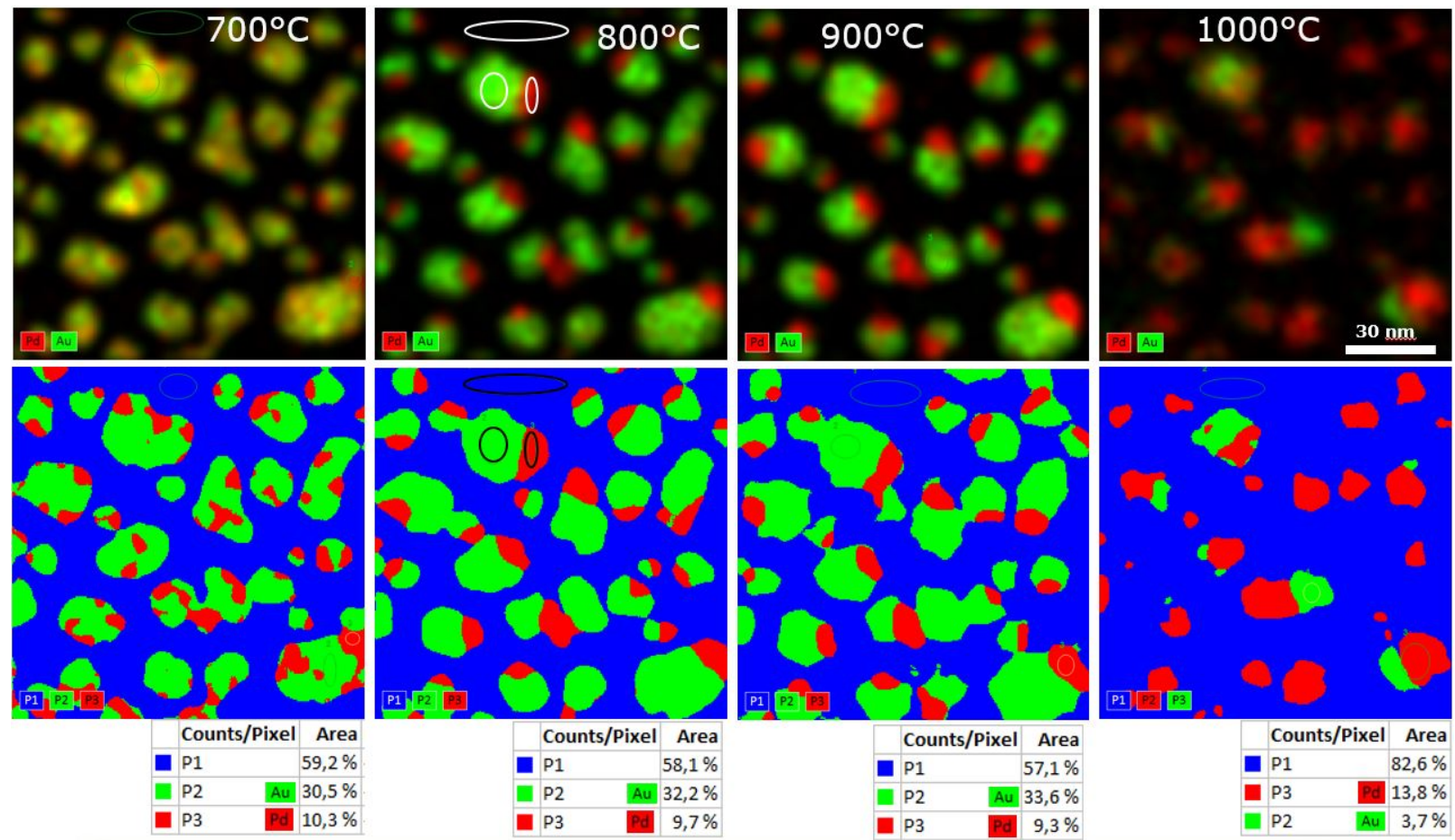

Figure 2. Element distribution and respective phase areal coverage at various temperatures. The regions used to define the three phases: exposed carbon film (P1), gold on carbon (P2) and palladium on carbon (P3) are marked in the second image. The tables below the images give the relative areal coverage of the identified chemical phases. Agglomeration takes place. Au starts to evaporate at higher temperatures, so its areal coverage is reduced at $1000^{\circ} \mathrm{C}$ while the exposed carbon film (P1) area is growing. The data show, how phase and particle analysis can be used to quantify in situ data. 